\section{> Protein structure through time}

Advances in time-resolved crystallography make it possible to follow ever more rapid protein structural changes.

A static structure of a protein gives a biologist much insight into its function, but researchers can gain an even deeper understanding by watching the protein in action. Detecting intermediate conformational states that exist only fleetingly, however, is a serious experimental challenge.

One approach used for studies of protein dynamics is time-resolved X-ray crystallography (TRX). In a TRX study, a laser 'pump' pulse is deployed to initiate a reaction, and then X-ray 'probe' pulses are applied to collect a series of diffraction patterns as the reaction proceeds. In the past, performing such experiments required highly specialized synchrotron sources. These instruments have a 'speed limit' of about 100 picoseconds, precluding experimental visualization of very fast protein conformational changes. But now, recent advances made possible by ultrafast X-ray free-electron lasers (XFELs),

\section{>>Precision optogenetics}

Optogenetic manipulation of neurons at cellular resolution holds promise for the dissection of neural microcircuitry.

The year 2015 marked the ten-year anniversary of the introduction of channelrhodopsin 2 (ChR2) into neuroscience. ChR2 has proven to be a powerful tool not only for stimulating neurons, but also for

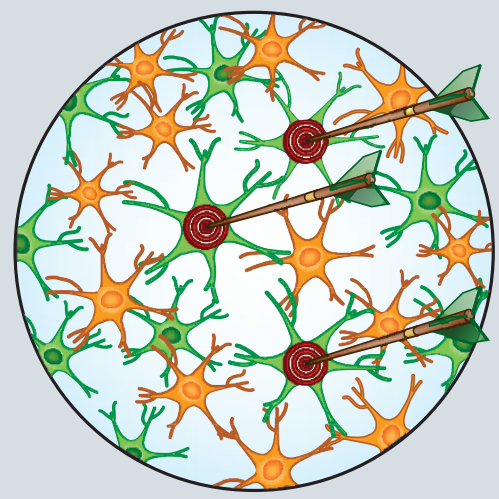

Targeting subsets of neurons within a genetically defined population.

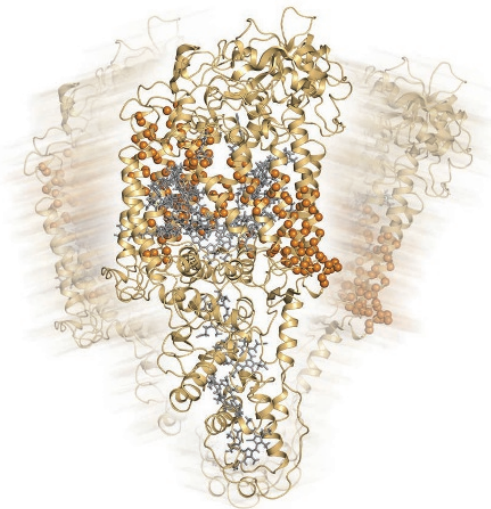

Time-resolved crystallography reveals protein dynamics. Image adapted from Nat. Methods 11, 923-926 (2014).

offering femtosecond-level time resolution, are allowing researchers to resolve very rapid structural changes that are undetectable by any other method.

In 2014, for example, researchers used XFEL-based TRX to follow the conformational changes that photosystem II undergoes as it catalyzes light-driven water splitting, at 5.5- ̊ resolution (Nature 513, 261-265, 2014). Later that year, a team used the approach to study the photocycle of photoactive yellow protein, resolving reaction intermediates at an impressive 1.6- $\AA$ resolution (Science 346, 1242-1246, 2014). And in 2015 , another group took advantage of ultrafast XFEL pulses to follow structural changes in myoglobin resulting from photolysis of the iron-carbon dioxide bond at its active-site heme (Science 350, 445-450, 2015).

Given the intense competition for XFEL beamtime at the very small number of instruments around the world, such experiments are even less generally applicable than a TRX experiment using a specialized synchrotron source. Thus a clever pumpprobe pulse-sequence approach that can be used to collect TRX data with any of the standard synchrotron beamlines, which are accessible to a much broader community, is a welcome development (Nat. Methods 11, 1131-1134, 2014). Though the technique's time resolution has not yet been demonstrated to be nearly as good as that of an XFEL, future advances may make this a possibility.

It will certainly be interesting to watch the TRX technique blossom, especially to see how researchers will adapt the approach to study ultrafast reactions in proteins triggered by stimuli other than light. Allison Doerr inspiring the development of other optogenetic tools. Typically, these tools are excited with wide-field illumination, and specificity is imparted by their expression in genetically defined neurons. But for a more detailed dissection of neural circuitry, patterned illumination schemes come in handy. These allow one to manipulate the activity of particular subsets of neurons within a genetically defined population.

The desired illumination patterns can be generated in different ways. Illumination with fast scanning mirrors is an option, or computer-generated holographic patterns may be produced with spatial light modulators. In addition, illumination schemes need to be integrated with the optical readout of neural activity that is often combined with optogenetic activation. Linking these two goals is not necessarily easy and requires optimization because of optical cross-talk between the optogenetic activators and the activity sensors.

Several recently introduced methods exemplify the various strategies available to researchers. Near-simultaneous illumination of neurons can be accomplished by focusing a two-photon laser to the size of a soma and switching between different neurons with the help of a fast scanning mirror (Nat. Neurosci. 17, 1816-1824, 2014). One can also achieve simultaneous illumination by splitting a two-photon beam into multiple beamlets and targeting them to the neurons of interest with a spatial light modulator (Nat. Methods 12, 140-146, 2015). In both studies, the use of a red-shifted optogenetic actuator (C1V1) reduced optical cross-talk with the green calcium indicators that reported neural activity. In freely behaving animals, fiberscopes can deliver one-photon illumination patterns generated in a similar manner (Neuron $\mathbf{8 4}$, 1157-1169, 2014).

Cellular-resolution optogenetics will continue to further our understanding of neural-subtype diversity and function. However, these approaches allow the stimulation of neurons in only a single 2D plane within the field of view so far, whereas neurons communicate and function in the $3 \mathrm{D}$ environment of the living brain. Expanding these optical stimulation approaches into three dimensions is likely to open up exciting possibilities for probing neuronal function. Nina Vogt 